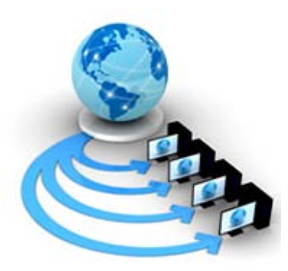

International Journal of Advanced Research in Computer Science

RESEARCH PAPER

Available Online at www.ijarcs.info

\title{
SIMULATIVE AND COMPARISION ANALYSIS OF LEACH AND HEED PROTOCOL IN NS2
}

\author{
Bushra Bano \\ Department of Electronics and Communication \\ CT Institute of Technology \& Research \\ Jalandhar, India
}

\author{
Joy Karan Shing \\ Department of Electronics and Communication \\ CT Institute of Technology \& Research \\ Jalandhar, India
}

\author{
Anurag Sharma \\ Department of Electronics and Communication \\ CT Institute of Technology \& Research \\ Jalandhar, India
}

\begin{abstract}
Nowadays, the wireless sensor network is the fast emerging technique for an ad-hoc network. Most of the routing protocol extended the network lifetime by uses the less consumption of battery by the sensor nodes. The proposed routing algorithms mostly use the hierarchical routing to increase the network lifetime and overcome the all issues in the network. The clustering approach is used to extend the lifetime of sensor node and decrease the energy consumption in the sensor network. These papers compare the various quality of service (QoS) parameter of network. In this paper we compare the LEACH and HEED protocol in term of QoS parameter. The experimental results show that the comparison of HEED protocol is better as compared to LEACH. Also the HEED algorithm is more energy efficient algorithm as compare to $\mathrm{LEACH}$, because this algorithm extend the network lifetime and decreases the energy consumption.
\end{abstract}

Keywords: WSN, LEACH, HEED, CH, BS

\section{INTRODUCTION}

. Wireless sensor networks (WSN) consist of a small sensor node. The sensors are small in size and less-complex device. These sensor nodes sense the signal and gather information from its neighborhood node. The gathering information is than forward from a wireless link through a multiple route pass to a base that utilizes it locally or associated with different system [1] [2].In wireless sensor network nodes are deployed in a large area. Sensor nodes required very low power. A sensor consists of a sensing unit, Processing unit, storage and power supply unit. The basic feature of sensor nodes is that it stores some information and processes this information for a transmission at the BS. The component is used for wireless sensor network are as BS, sensor node and the interferences between the user through internet. In addition sensors are the most important component of the network. The applications of wireless sensor network are used in the military application, Area Monitoring, Environmental application and weather forecast detection. The nodes deployment can be fixed, regular and mobile [3][5].In a WSN the communication can be done using either single hop Or multiple hop. In single hop communication only one hop is used to transmit the signal or information from sensor node to BS. In the multihop communication two or more hops is used to convey the information from a cluster head to destination [14]. During multihop communication the information is not directly Transfer so that the network is divided into cluster. So clustering approach is used in each cluster. In clustering approach sensor transfer information to cluster head aggregate information that are received from the different nodes in a cluster. When the all information is gathering the cluster head transmit to the Base station. The sensor node can be homogeneous or heterogeneous in the network. In homogenous network same type of node is used where as in heterogeneous network different type of node is used i.e. Active node or sleep node. The routing techniques can be classified into three categories i.e. flat, hierarchical and location based. In the Flat routing all node work together to collect the information and route this information in the network. In this routing technique the function of node is identical. In location based routing the information is sense on basis of node location. After that the node build the optimal routing path for the transfer of information on the basis of each node information .In the hierarchical routing the cluster head is used to collect the information and route the information hop by hop form the downstream(member nodes) to upstream(CH)[3] [16]. The hierarchical divide into four categories i.e. Chain Based Data Aggregation, Grid Based Data Aggregation, Tree Based Data Aggregation, and Cluster Based Data Aggregation. In chain based all nodes knows the information of another node to create a chain for the transmission of information. In the Grid based data aggregation the information is transmitted on the basis of geographic position where the aggregators transfer the information. In this approach node does not communicate with each other only the aggregator nodes communicate. In the tree based data aggregation the network is form into tree to aggregate the information. In the cluster based data aggregation the network is divided into cluster. Each clusters form its own cluster head to aggregate the information. The cluster head transfer the information to the base station [16]. LEACH protocol is based on MAC Protocol. This algorithm distributes energy consumption evenly in all sensors node of the network. In this algorithm the network are divided into 
cluster. In each cluster one cluster head is selected on the basis of probability. The energy factor is important in clustering so that sensor node with higher energy selected as a cluster head random rotation. LEACH protocol is work in two phases; Setup phase and the Steady state phase. In the setup phase In the set-up phases sensor nodes want to be cluster head on the basis of random number between 1 and 0 and selected the value of node is less than the threshold value that node number is than compare with the threshold value. After that select as a cluster head for the next round. In the steady state Phase all the data is transmitted from the sensor node to cluster head and then cluster head aggregate the data to transfer at the base station [12] [13]. In HEED (Hybrid Energy- Efficient Distributed Clustering) two parameter are used, the first parameter is residual energy and the second parameter is network topology (distance between the neighbors node, node degree) [4]. The operation of HEED is divided into several phase. In the initialize phases the node defines its probability to become a cluster head. In which the sensor select their probabilities between 0 to 1 . In the main phase the some node become a tentative node and rest of node are member in cluster. In final phase the least communication cost of node become $\mathrm{CH}$ [11].

\section{RELATED WORK}

Kaur H., et al. [2010] proposed the H-HEED (Heterogeneoushybrid energy efficient distributed) Algorithm to extend the network lifetime. In this algorithm the different level of heterogeneity impact is describe in term of node energy. The simulation result show that the H-HEED algorithm is better as compare to HEED in term of lifetime and more effective data packet transmission [15]. Ren F., et al. [2011] implements Energy-Balanced Routing Protocol (EBRP). In this algorithm the forces is apply on the packets to pass in the direction of BS through the dense area with very low residual energy. The results show that this algorithm work more effectively as compare to other exiting routing protocol in term of extended network lifetime, energy balance, and coverage ratio [7].Sharma S., et al. [2013] presented a survey regarding to the routing protocol, architecture design issue, power consumption and the communication capabilities. For implement the networks the some factors are considered such as the mobility, coverage area, , power consumption, communication capabilities etc. In this paper also describe some issues for WSNs like as security, energy consumptions, network lifetime [8]. Mardini W., et al. [2014] proposed R-HEED protocol to extend the lifetime of network and residual energy. This algorithm selects the cluster head on the basis of turn schedule. In this algorithm if the energy of current cluster head is goes down under the threshold value, than transmitted the reclustering message to the BS. The base station transmitted the re-clustering message among all the nodes to select the cluster head for the next round. Otherwise re-clustering is not required for the next round. The simulation result show that the RHEED perform better as compare to HEED protocol [9]. Varma A., et al. [2015] suggested the entire network is divided into a cluster, and multipath are restricted to these clusters by which traffic will be distributed only to the cluster without propagation entire network and does not restricted and does not cause delay, energy consumption, increase delivery ratio between the nodes. Multi-path routing provides an easy mechanism to distributed traffic, balance networks load and fault tolerance [10]. Eshaftri M., et al. [2015] implement LCP protocol to extend the network lifetime of wireless sensor network. In this paper algorithm use the inter cluster approach which balance the energy consumption in the network. This algorithm rotates continuously to select Cluster Head $(\mathrm{CH})$ with higher residual energy in each round. The results show that this approach extended the network lifetime and also effective for balances the energy consumption among all sensor nodes [3].Singh S., et al.[2015] presented a survey paper on cluster based routing protocol in wireless sensor network. In this paper the various routing algorithm is presented in term of the scalability, energy efficiency, cluster stability, delivery delay, load balancing and algorithm complexity. Also the merits and demerits of clustering algorithm is define. The comparison between various routing technique shows that the cluster based routing algorithm improve the performance of wireless sensor network [6]. Ma Z., et al. [2016] presented the improved version of LEACH algorithm known as LEACH-C (Low Low -Energy Adaptive Clustering Hierarchy-centralized). In this paper energy is distributed in the network by changing the nodes into cluster head. This algorithm balances the energy efficiently as compare to LEACH algorithm. The simulation result show that improved version of algorithm can extend the lifetime of the system [11].

\section{SIMULATION SETUP}

The network is simulated in network simulator NS2. We considered the heterogeneous sensor network with 41 sensors nodes that are deployed evenly distributed in the $800 \mathrm{~m} * 800 \mathrm{~m}$ area. In which the one node is used as the sink (base station).We analyzed and compared the LEACH protocol and HEED protocol with each other. Table: - 1 show the network configuration used in the NS2.

Table 1: Network configuration

\begin{tabular}{|c|c|}
\hline Parameters & value \\
\hline Deployment field & $800 * 800 \mathrm{~m}$ \\
\hline Protocol & LEACH,HEED \\
\hline No. of nodes & 41 \\
\hline Simulator & NS2(network simulator 2.35) \\
\hline Packet size & 1000 bytes \\
\hline Antenna type & Omni antenna \\
\hline
\end{tabular}


The Figure 1 shows that the 41 node are deployed in a network area. The figure 2 shows that the nodes transfers the information and aggregate the information from its neighboring nodes and cluster head. After the aggregation of information the cluster head communicate with base station for the transfer of information.

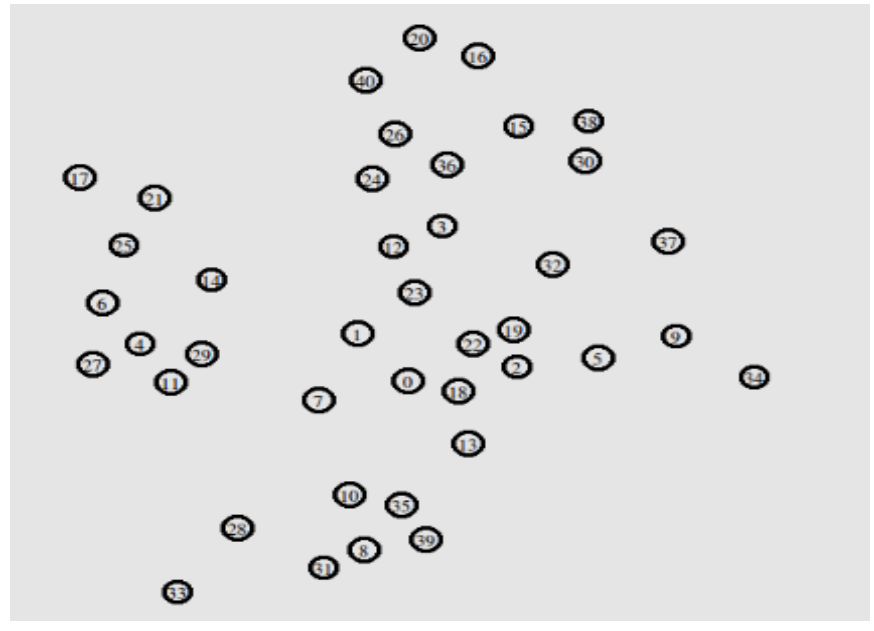

Figure 1. Network Deployment

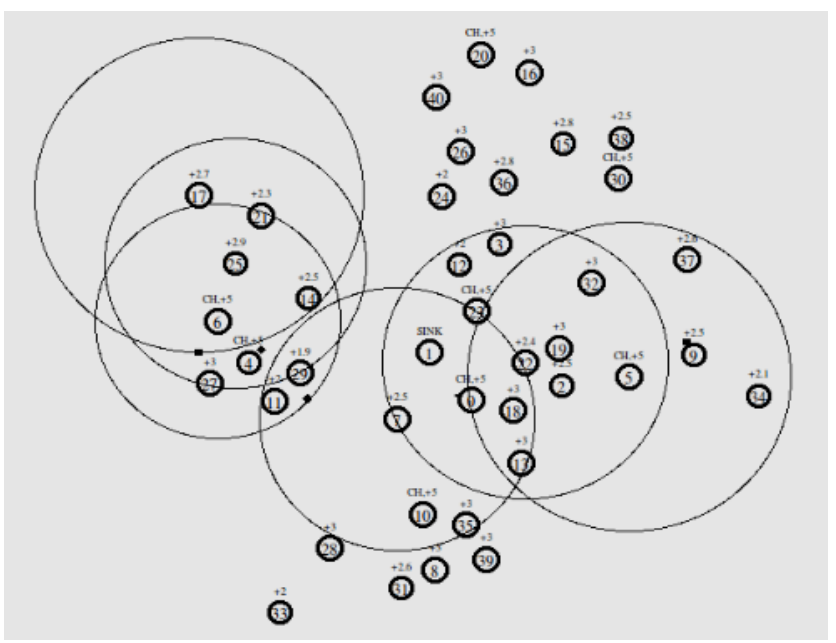

Figure 2. Data Aggregation and Data Transfer at Base Station

\section{RESULT \& DISCUSSION}

We analyze the LEACH and HEED Protocol with each other. For comparison we use some parameter such as delay, packet loss and throughput. The result show according to the simulation time for $800 * 800 \mathrm{~m}$ area. In $8000 * 800 \mathrm{~m}$ network area 41 nodes are deployed random manner. Figure 1 shows that the delay comparison result of LEACH and HEED protocol. The delay is more in LEACH as compared to the HEED protocol. The more time taken by the packet for the transmission in the network from the cluster head to sink is known as delay. Figure 2 shows that the packet loss in LEACH and HEED. The packet loss is high in LEACH protocol as compared to the HEED protocol. If the packet is not successfully received at the destination is known as the packet loss. In packet loss the packet are drop during the transmission. The packet loss is more in LEACH because in this protocol the re-clustering is not done. Figure 3 show that the throughput of the HEED protocol is higher than as compare to the LEACH protocol. The maximum amount of the successfully information is received from source to destination in a particular time is known as the throughput. The comparison result show that the performance of HEED protocol is better as compare to the LEACH protocol.

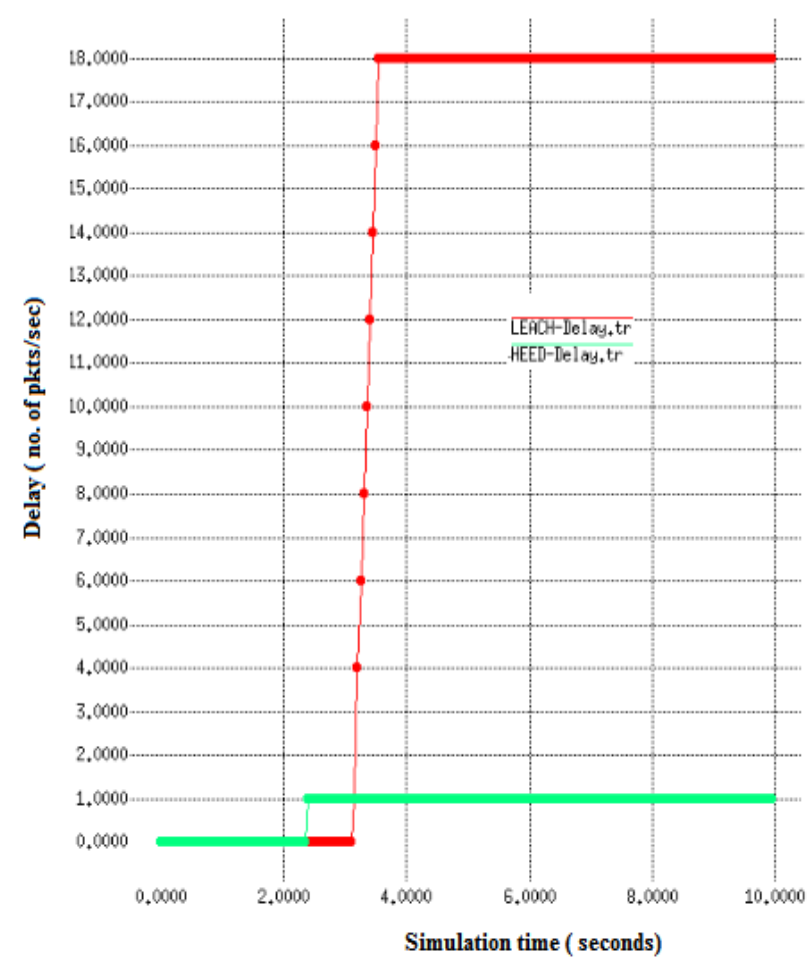

Figure 3. End- to- End Delay

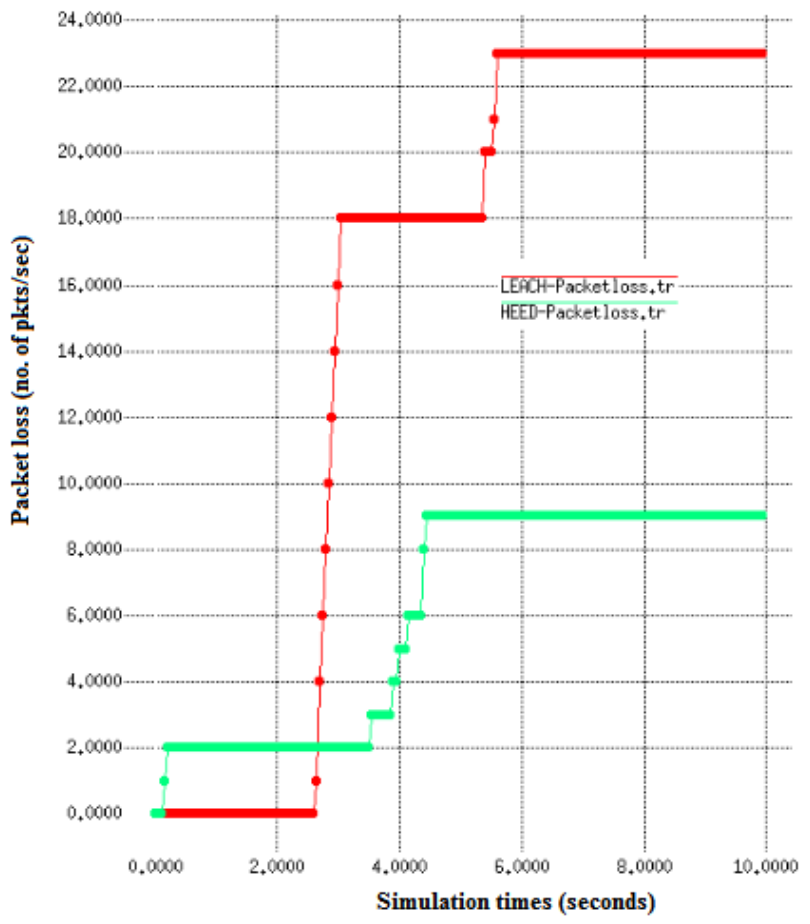

Figure 4. Packet loss 


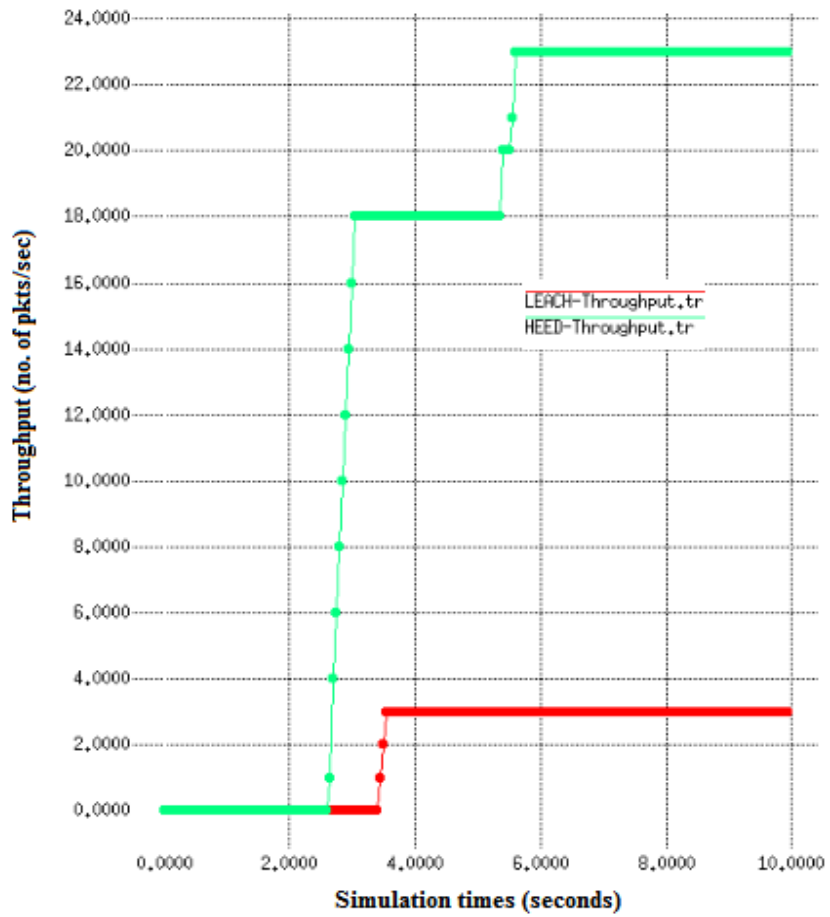

Figure 5. Throughput

\section{CONCLUSION}

The various clustering protocol are used to extend the network lifetime and also balance the energy consumption of WSNs. In this paper we compared the LEACH and HEED protocol by using different parameter. The simulation result show that the HEED protocol is better as compare to LEACH protocol in term of packet loss, delay and throughput. But still there are some issues in WSNs such as reliability, scalability etc. So that to overcome this issues further enhancement is required for this protocol.

\section{REFERENCES}

[1] Lee j., Jung k., Lee D., “The Routing Technology of Wireless Sensor Networks Using the Stochastic Cluster Head Selection Method", International Journal of Control and Automation (IJCA) Vol. 8, No. 7, pp 385-395., 2015.

[2] Vashist S., Soni M.K., Himanshi, "Study of Wireless Sensor Network Using LEACH Protocol”, International Journal of
Innovative Technology and Exploring Engineering, vol.3, 2013.

[3] Younis O., Fahmy S., "HEED: A Hybrid, Energy-Efficient, Distributed Clustering Approach for Ad Hoc Sensor Networks,” IEEE, vol. 3, No. 5, 2004.

[4] Hasan S., Husain Z., Shing R.k., "A Survey of Wireless Sensor Network", International Journal of Emerging Technology and Advanced Engineering Volume 3, Issue 3, 2013.

[5] Singh S., Sharma S.C., "A Survey on Cluster Based Routing Protocol in Wireless Sensor Networks", International Conference on Advanced Computing Technologies and Application (ICACTA), pp 687-695, 2015.

[6] Ren F., Zhang J., He T., Lin C., Das S., "EBRP: EnergyBalanced Routing Protocol for Data Gathering Wireless sensor network", IEEE TRANSACTIONS ON PARALLEL AND DISTRIBUTED SYSTEMS, Vol. 22, No. 12, pp 21082125, 2011

[7] Sharma S., Mittal P., "Wireless Sensor Networks: Architecture, Protocols", International Journal of Advanced Research in Computer Science and Software Engineering, Vol. 3, Issue 1,pp 303-308, 2013.

[8] Mardini W., Yassien M.B., Khamayseh Y., B. A. Ghaleb., "Rotated hybrid, energy-efficient and distributed (R-HEED) clustering protocol in WSN," WSEAS TRANSACTION on COMMUNICATIONS, vol. 13, pp 275-290., 2014.

[9] Varma A., Reddy A., Y Theja., R., Arunkumar T., "Cluster Based Multipath Dynamic Routing Protocol for Wireless Sensor Networks", Indian Journal of Science and Technology, Vol. 8, pp 17-22., 2015.

[10] Ma Z., Li G., Gong Q., "Improvement on LEACH-C Protocol of Wireless Sensor Network "International Journal of Future Generation Communication and Networking Vol. 9, No. 2, pp 183-192.,2016.

[11] Reshma I. Tandel, "LEACH Protocol in Wireless Sensor Network: A Survey" International Journal of Computer Science and Information Technologies, Vol. 7 (4), pp18941896, 2016.

[12] Malik M., Singh Y., Arora A., “Analysis of LEACH Protocol in Wireless Sensor Networks", International Journal of Advanced Research in Computer Science and Software Engineering, Vol. 3, Issue 2, pp 178-183, 2013.

[13] Chand S., Singh S., Kumar B.,“ Heterogeneous HEED Protocol for wireless sensor network”, Wireless Pers. Commun , DOI : 10.1007/s 11277-014-1629-y.

[14] Kaur h., Sharma A., "Hybrid Energy Efficient Distributed Protocol for Heterogeneous Wireless Sensor Network", International Journal of Computing Application, vol.4, No. 6, 2010.

[15] Parmar R., Dr. Kumar S., "A Survey: Cluster Based Routing Protocol in Wireless Sensor Network”, International Journal of Advanced Research in Computer Engineering and Technology (IJARCET), Vol. 4, Issues 10, 2015 